\title{
Design and Implementation of Energy Saving Control and Regulation System for Metallurgical Furnace
}

\author{
ShuWen Chen, YongShen Chen, and WeiWei Guo \\ \{ chenshuwen@126.com\} \\ School of materials and metallurgy University of Science and Technology Liaoning Anshan 114051, \\ China
}

\begin{abstract}
This paper from the perspective of saving fuel gas, reasonable design of temperature control system. The thermostat is designed S7-200 series PLC adoption. The communication method of the computer and programmable controller (PLC) temperature control system is described. Software part of the application of the PLC principle to achieve system control. Hardware part of the temperature sensing element thermocouple temperature field, flow meter, fuel flow regulation, adjusting the thermocouple output electrical signal supply line for comparison with the given values, if there is a difference, the difference through the operational amplifier and issued a directive to the actuator, the actuator to perform the task. The simulation interface of the application configuration, virtual reality condition, the master-slave real-time monitoring system composed of computer and PLC to give full play to the their respective advantages in the industrial control, realize the decentralized control and centralized monitoring and other new features. Write part of the program, to research on energy efficient and reasonable utilization as the theme, combined with typical furnace, energy for metallurgical furnace the huge energy consumption equipment rational utilization and effective control method is put forward to improve the reasonable design of the program, resulting in applications get effective implementation, for enterprises to save energy, create economic benefits to contribute.
\end{abstract}

Keywords: PLC; control; computer; configuration technology.

\section{Introduction}

So far the majority of domestic metallurgical furnace is built in the last century, the control system configuration has been outdated. There are quite a few or by the dial shows the base type instrument control, in seeing the level meter, a manual control valve, controlled by a computer operating system has not been scientific and reasonable application, as soon as possible to change the backward situation, the effective measures is one of the application of modern computer control technology, according to the requirements in the heating process of automatic processing of metallurgical furnace fuel supply, carries on the design to the metallurgical furnace fuel monitoring and control system. The main purpose of this paper is the energy-saving heating furnace control system design and implementation [1], that is, through the control of furnace temperature to control the amount of fuel and air supply, to achieve the purpose of saving energy, so that the field operations staff is easy to grasp. The 
heating condition of metallurgical furnace is a typical complex industrial process control system, which has many characteristics of complex system. For example, the difficulty of feature modeling, severe interference, multi variable, time varying, nonlinear, large hysteresis and inertia. Therefore, the heating furnace is a major problem in industrial automation. In all types of fuel, fuel gas combustion can be most easily controlled, usually metallurgical furnace using natural gas, the highest combustion efficiency, combustion process experienced three stages: mixing, preheating ignition, gas and air combustion. Previous metallurgical furnace control mode is mainly to control the furnace temperature, by adjusting sensors and actuators were automatically adjusted according to set an air and gas mixing ratio, usually cases, operators in the set up of furnace temperature more than high volume, fluctuations in heating condition, and easy to cause oxidation burning loss of metal, thereby reducing the heating quality, and leads to the increase in energy consumption. When the excess air coefficient and the empty burning than the set value, the control of natural gas flow can make the combustion air volume constant also control the temperature constant. The design of the metallurgical furnace temperature control adopted limiting control mode, namely according to the given air and gas ratio, according to the variation of temperature. At the same time, science reasonable regulation given fuel flow, in order to ensure the temperature regulating process, the fuel and air are according to the design given the ratio of gas supply. This can not only save energy, but also to prevent environmental pollution, to bring positive energy to the enterprise and the environment. This paper, starting from the point of view of saving fuel gas, application of PLC module of furnace temperature control system for a reasonable design, gives the application, is effective in the field of energy research, reasonable use is a tenet, with the typical type of furnace, energy for metallurgical furnace the huge energy consumption equipment rational utilization and effective control method is put forward to improve the reasonable design of the program, resulting in applications get effective implementation, for enterprises to save energy, create economic benefits to contribute [2].

\section{Analysis of Metallurgical Furnace Control System}

Metallurgical furnace has many kinds, milk steel heating furnace is one, now heating furnace as an example to design out under the precondition of satisfying the requirements of the production process, the reasonable control of the air fuel ratio, save fuel consumption, is today's metallurgical furnace optimization design of the basic requirements. As is known to all, the heating furnace is a complex thermal equipment, by the metal heating furnace, fuel supply device and combustion device for wind and discharge device, waste heat reuse equipment, vaporization cooling device, thermal parameter detection and automatic control device. Natural gas is a colorless, slightly rotten smell of gas, lighter than air, the density in the $0.73 \sim 0.80 \mathrm{Kg} / \mathrm{Nm} 3$ range. Once the fire is easy to ignite, in the range of 64 85 degrees Celsius, is the ignition temperature range. Natural gas under the jurisdiction of the shooting ability strong, flame to pale blue, very bright is because it in the combustion itself contains a hospital, hydrocarbons in case of high temperature and decomposition, precipitated a solid carbon particle sake. 


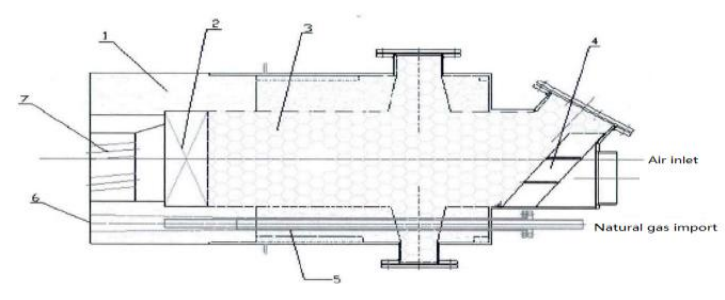

Fig. 1. The burner system structure diagram

\subsection{Heating System of Metal}

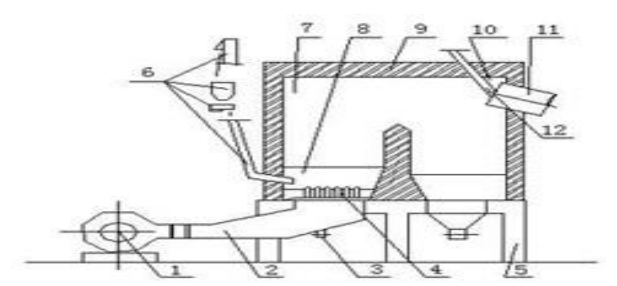

Fig. 2. Schematic diagram of metallurgical furnace structure

The heating system is set up to meet the heating conditions, and the heating method is adopted. The heating system including temperature and strength of the system and heating system. Refer to the cross section of the furnace space segment of the temperature distribution in the continuous heating furnace temperature system; heating system refers to the boiler refers to the furnace heating ratio. The temperature system is a basic; the heating system is the guarantee of the temperature system. All enterprises are given in the operation temperature system, namely each temperature requirements. The three section heating system of working condition system is the first choice of the design, the three section also known as: preheating section, heating section and hot section. Namely: the metal in different regional temperature preheating, heating, heat heating period. Metallurgical furnace engineering practice proved that the heating system is more perfect and reasonable, metal initially in the low temperature region in the preheating section in the slow heating preheating temperature of force is small, not easy to cause heating defects (cracks). When the metal temperature across the 500 600 degrees Celsius, into the plastic zone, then you can quickly heat up, until the heating period [3].

\subsection{Control System Design}

To correct the zero point and range, use the transmitter before the first work. The purpose of adjusting: enable output tension limit $4 \mathrm{~mA}$ and corresponding measurement lower limit value, relative to the input and output characteristic curve of horizontal movement. The analog transmitter can adjust the potentiometer through its zero point adjustment. The purpose of adjusting the range: to change the slope of the characteristic curve. Is the output of the transmitter, the upper limit of the value and the measured value of each other, which can be adjusted by analog transmitter on the range adjustment potentiometer. In a simple process 
control system, it consists of four parts: controller, transmitter, actuator and controlled object. The output of the transmitter (or detecting element) will generally increases output signal proportional to be detected variables (controlled variable), such as: temperature increased, DDZIII type pressure transmitter current output also increased. So the detection or change of the link is generally positive. When the deviation is increased, the output of the controller is also increased, the controller has a positive role in promoting, and vice versa for the negative effects or negative effects. When the actuator is an electric valve is generally positive effect, because its output valve opening will with the increase of the input control signal and increase, but if used pneumatic diaphragm control valve, due to the sub valve to open the gas valve and gas off valve, gas valve opening for positive effect, air to close valve for the reaction. Relative humidity control, if manipulated variables increase, the control of tension temperature also increased, is a positive role. Otherwise, it is negative. Therefore, a simple process control system to achieve a negative feedback control [4].

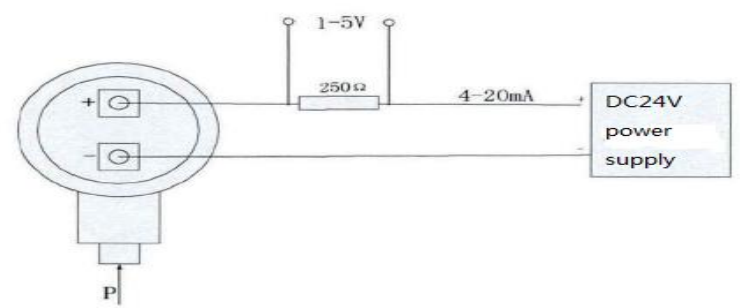

Fig. 3. Two wire transmitter current and voltage conversion wiring diagram

\subsection{PLC Analog Control}

Using PLC as a controller, the time or numerical simulation is a continuous physical quantity. For example: temperature from $0 \sim 1200$ degrees $C$, which is analog. In a process control system, the physical quantity of temperature is changed to the voltage or current signal by the detection of the transmitter (or sensor). Because the PLC program can only operate on the number of digital data, the measurement of the voltage or current signal is also measured to analog digital conversion (A/D). PLC operating results if you want to make the output signal to control the implementation of the agency's action, such as: electric control valve opening from 0 ? $100 \%$ change, it is required to convert the digital quantity into voltage or current form analog signal (D/A). For large inertia temperature of metallurgical furnace system. In PID regulation, professional knowledge and the parameter setting of tension set higher experience is required, if the parameter is not good also difficult to obtain good regulating effect, so it is more complex. For some larger temperature system, the temperature control system is formed by using industrial control machine and PLC, which is corresponding to the temperature conversion module.

\section{System Design and Implementation}

With the development of electronic technology, the programmable logic controller (PLC), now has the original single logic by volume control, gradually transition to have function of computer control conditions, and implement and PC communication, continuity of complex control is accomplished. At this point, PLC in the process of control and regulation of metallurgical furnace, play a very important role, can be together with the computer to form a 
complete control system. Therefore, it has important practical significance to design a temperature control system which is reasonable and practical. So far in the metallurgical industry, the precise control of temperature is a major problem for the enterprise, to solve this problem, the computer real time monitoring has the superiorities of simple programming, controllable force strong, high reliability, flexible configuration, convenient application and easy expansion, in many metallurgical furnace control system, has become today and in the future of furnaces in metallurgical industry control domain development direction. PLC on the calculation of data, processing, user interface and other functions of the management ability is poor, hinder the temperature change of the furnace at any time monitoring and tracking. However, the computer in this area will be able to protect the PLC this deficiency. In the temperature control, the PLC as the next bit machine, the implementation of on-site monitoring, the computer as the host computer, to deal with information. The communication circuit is used to exchange and transfer information between PLC and computer. Using computer and PLC constitute the main, from monitoring condition, can better play their respective strong in the furnace temperature control, decentralized control and centralized supervision. This design uses the Siemens S7-200 series PLC, PLC serial communication with the computer connection, stable operation can obtain monitoring interface is friendly. Enter a furnace temperature of each segment of the given value in computer, dynamic display of baked temperature change from time to time, the real purpose of the real-time monitoring of the temperature [5].

Configuration control technology is a kind of computer control technology. Using the configuration control technology of computer testing and control system and the tension of general computer measurement and control system in the structure of no essential difference. They are made up of controlled object, sensor, I/O interface, computer and executive mechanism. The function of the sensor is to detect various parameters of the controlled object. Through the sensor, the computer can "perceive" the production of the situation, the parameters on the display display. According to the deviation between the actual value of the parameter and the setting value, the control command is issued according to a certain control algorithm, and the action of the executing mechanism is controlled to accomplish the control task. Such as the water tank water level control system in computer by the water level sensor measuring know the height of the water level or lower limits, will the situation shown on the display, and according to the water level control valve closing and opening to achieve the purpose of water level measurement and control. Sensors, actuators are generally placed in the production site, and charged with the object together, also called the field equipment. Using the configuration technology of the system, the computer is generally placed in the control room.

\section{Conclusion}

The purpose of this design is to save energy for metallurgical furnace, so in the actual application of computer monitoring interface on the tension of a metallurgical furnace furnace for real-time monitoring and regulation, the S7 - 200 series PLC control system according to the three sections of the heating furnace operating parameters design according to the value of the furnace temperature fluctuation and the corresponding adjustment of natural gas and air flow adjustment scheme of the. So that the operators can be far away from high temperature, the danger, the noise of the working environment, scientific and reasonable operation according to the setting of computer operating value, to avoid the due to the operation level of personnel, work attitude different while the temperature is not reasonable control, and the 
effect of metal heating quality but also a waste of energy, but also bring pollution to the ecological environment.

\section{References}

[1] Shi Yanpeng, Qi Xiangdong. Mechanical engineering and automation of boiler level control system based on configuration software. No. 05. (2011)

[2] Cai Jun, Cao Huiying. Design and research of temperature control system based on. PLC microcomputer information. No. 04. (2013)

[3] Dai Xing, Xie Shouyong, He Binghui, Guan Ping. Research on greenhouse control system based on. PLC Agricultural Mechanization Research. No. 01. (2014)

[4] Wan Neng Yang, Fu Jun Qin. Based on the PLC network temperature monitoring system. Xihua University Journal (Natural Science Edition). No.06. (2012)

[5] yellow column deep, Huang Chaolin. High precision temperature control system based on. PLC mechanical and electrical engineering technology. No. 02. (2011) 